Musées, Patrimoine et Culture scientifiques et techniques

$162 \mid 2015$

novembre-décembre 2015

\title{
Interroger la relation entre public, institutions culturelles et numérique
}

Camille Jutant

\section{OpenEdition \\ Journals}

Édition électronique

URL : http://journals.openedition.org/ocim/1578

DOI : 10.4000/ocim. 1578

ISSN : 2108-646X

Éditeur

OCIM

Édition imprimée

Date de publication : 1 novembre 2015

Pagination : 15-19

ISSN : 0994-1908

Référence électronique

Camille Jutant, «Interroger la relation entre public, institutions culturelles et numérique », La Lettre de I'OCIM [En ligne], 162 | 2015, mis en ligne le 01 novembre 2016, consulté le 30 avril 2019. URL : http:// journals.openedition.org/ocim/1578; DOI : 10.4000/ocim.1578

Ce document a été généré automatiquement le 30 avril 2019

Tous droits réservés 


\title{
Interroger la relation entre public, institutions culturelles et numérique
}

\author{
Camille Jutant
}

La base JOCONDE, première base de données regroupant les peintures des musées français : www.culture.gouv.fr/documentation/joconde/fr/pres.htm

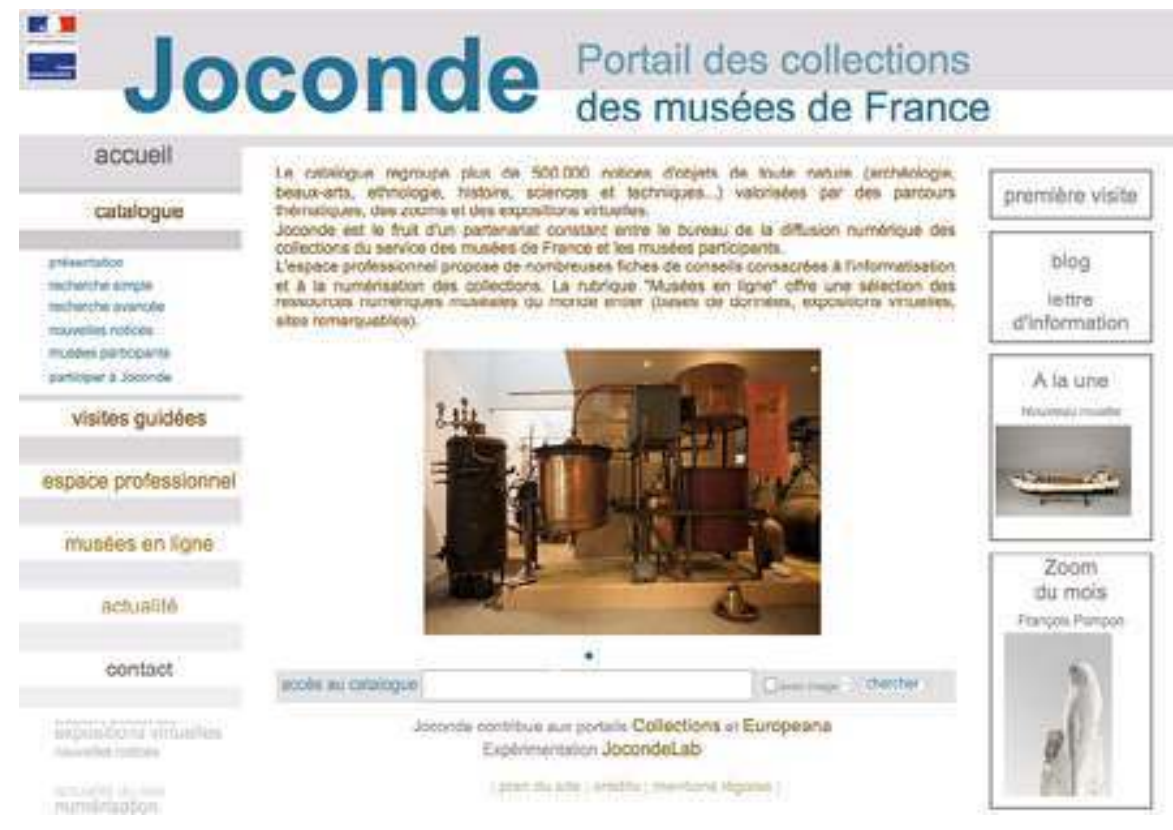

(c) DR

1 Les musées se sont engagés sur le terrain des technologies numériques depuis presque quarante ans et pourtant les discours prophétiques sur la révolution numérique toujours en cours, toujours en train d'advenir, continuent de produire une injonction à la nouveauté dans la sphère muséale (Jeanneret, 2011 ; Sandri, 2012 ; Wievorka, 2013). En 
réalité, la relation entre technologies numériques, institution muséale, collections et visiteurs peut être comprise comme la lente maturation d'un ensemble d'enjeux liés aux missions du musée, depuis qu'il existe dans sa forme contemporaine, c'est-à-dire des enjeux d'accès à la culture, de circulation des savoirs et de définition de ce qui constitue une expérience culturelle. De ce fait, on ne peut pas essayer de comprendre les relations entre musée et numérique sans regarder la trame de fond des discours politiques, médiatiques et promotionnels sur le rapport entre numérique, publics, pratiques et musées. Par l'analyse de ces discours, on comprend que les bénéfices liés au numériques avant d'être une réalité observable - sont les artefacts d'une pensée sur le renouveau des relations entre institutions et grand public ainsi que du renouveau d'une forme de démocratisation culturelle.

2 Nous pouvons citer au moins deux exemples. Les bénéfices attendus de la relation entre numérique et musée sont tout d'abord le fruit d'une injonction à l'adaptation, à la modernisation de l'institution (on en trouve de multiples exemples dans les appels à projets qui financent la production de dispositifs de médiation ${ }^{1}$ ou les rapports d'activités des musées eux-mêmes qui déclarent vouloir s'engager sur le terrain de l'innovation). Il est question de «nouveaux» paradigmes communicationnels. La notion d' « interactivité » participe, à ce titre, au premier chef, à la construction de la norme idéale à atteindre en matière de communication (Proulx et Sénécal, 1995 ; Le Marec, 2007 ; Brochu et $\mathrm{al}, 1999)$. Une autre injonction est celle qui consiste à répondre enfin à des enjeux de politique culturelle qui ne sont pas nouveaux. Parmi tant d'autres, l'enjeu de la diversité des publics, par exemple, se trouve réactivé par la représentation associée aux usagers des technologies, en l'occurrence bien souvent les jeunes, les digital natives, qu'il s'agirait de faire venir dans les lieux de culture par la promesse d'une familiarité avec ces outils, comme en témoigne cet extrait: «aller sur ces outils, c'est aller au devant des publics sur les espaces qu'ils occupent durant la majeure partie de leur temps de navigation $»^{2}$.

3 Cet article ne peut rappeler tous ces enjeux ni explorer toutes les questions soulevées par les discours sur les relations entre musée et numérique. Néanmoins, il propose tout d'abord de rappeler brièvement que ces enjeux se sont trouvés réactivés au gré de l'apparition successive de différentes générations d'outils, de services, de solutions technologiques. Il propose ensuite de prendre un exemple d'enjeu, celui de l'accès, pour montrer comment s'enchâssent les problématiques et les questions. Le point de vue choisi sera celui des relations entretenues entre les dispositifs numériques et les publics. Il ne traitera donc pas des enjeux de collection, de conservation, ou encore de modèles économiques, ou de modèles professionnels, que ne manque pas de soulever l'entrée du numérique au musée, mais du statut de ces dispositifs numériques qui offrent de repenser la question de l'accès au musée et de celui des publics-internautes qui semblent engagés dans une expérience culturelle spécifique.

\section{Petite archéologie des dispositifs numériques au musée}

4 Il est courant de lire ou d'entendre que les musées viennent tout juste de s'ouvrir au numérique et qu'il s'agit même d'une révolution. Si la présence du numérique s'intensifie au musée comme dans le reste de la société, elle est loin d'être nouvelle. Il ne s'agit pas ici 
de faire l'histoire des dispositifs numériques au musée, mais je voudrais rappeler qu'on pourrait au moins faire remonter cette histoire aux années 1970 et surtout que l'on pourrait avoir à l'esprit que chaque génération de dispositifs numériques a été porteuse d'un certain modèle de médiation et d'une certaine image de la relation entre publics et musées.

Jeu vidéo Versailles 1685 : Complot à la cour du Roi Soleil (éditeur : Cryo Interactive Canal + Multimedia, Développer, Cryo Interactive, Canal + multimédia et Réunion des musées nationaux)

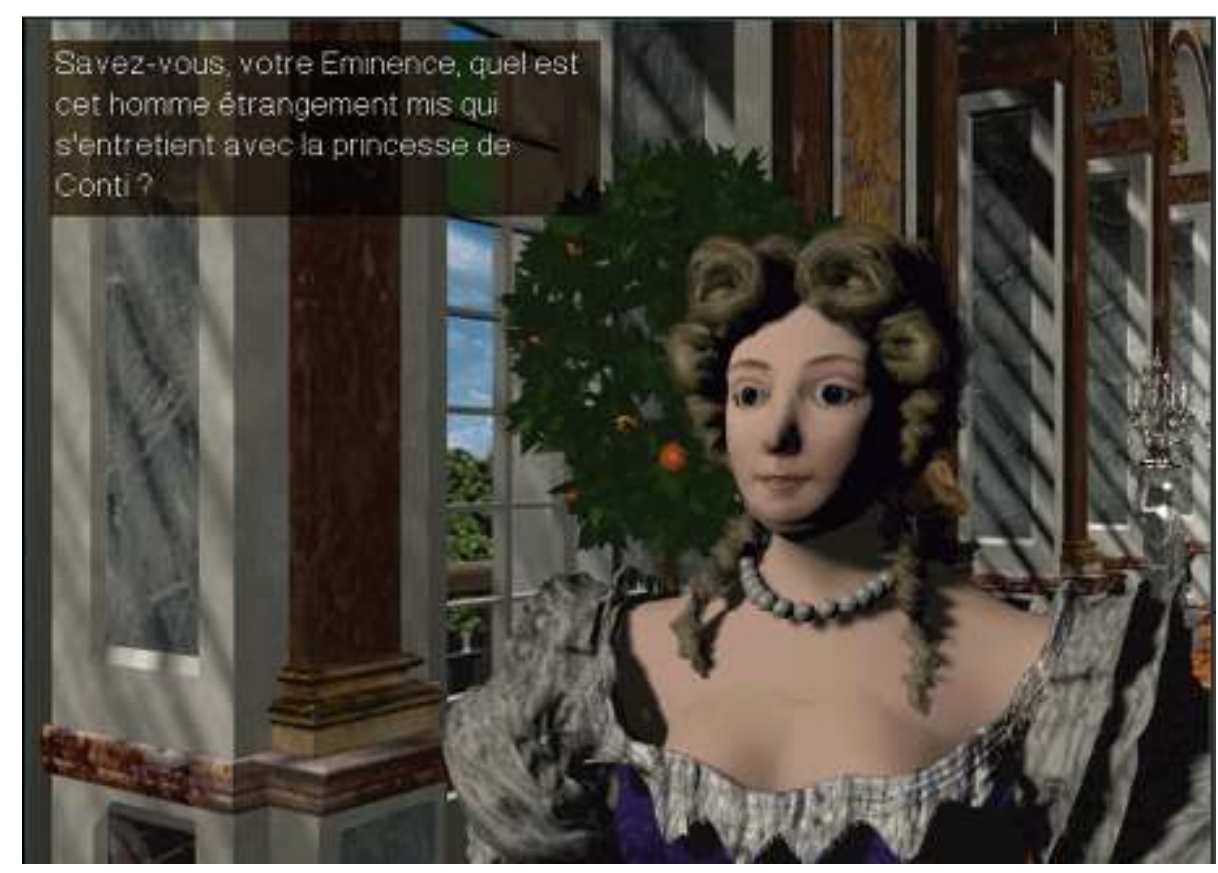

(c) DR

Pour mémoire, en France, la création de la première base de données, intitulée «Joconde », regroupant les peintures des musées français date de 1975. En 1986, les premiers postes de consultation numérique sont installés à destination des visiteurs de la Cité des Sciences et de l'Industrie, ainsi qu'au musée d'Orsay. Cette époque qui marque le début d'un vaste programme de numérisation des collections des musées de France, soutenu par la mission Recherche et Technologie, ouvre la voie à une réflexion sur la dimension virtuelle du musée. La reproduction de l'œuvre, qu'elle ait une fonction d'inventaire ou de consultation, produit une économie du double, et ainsi interroge les notions d'accès, d'ubiquité et d'authenticité (Desprès-Lonnet, 2009). Abraham Moles rappelle que la recherche de la haute fidélité ou de la haute définition n'est pas sans évoquer le mythe de la recréation à l'identique. Des chercheurs comme Daniel Jacobi (2005), Olivier Le Deuff (2007) ou Eva Sandri (2012) ont montré que cette numérisation soulevait des enjeux cruciaux pour l'identité des documents et des représentations des œuvres. Un autre ensemble de dispositifs apparait à la fin des années 1980. Ils utilisent la numérisation des objets et des espaces, mais permettent de surcroît la reconstitution d'espaces, vestiges ou bâtis disparus, bien souvent considérés comme patrimoniaux ${ }^{3}$. Pour exemple, en 1988, les dispositifs « Mémoires de pierre » et « Gunzo » montrent des images de synthèse et la reconstitution de l'abbaye de Cluny. La mise en scène du patrimoine, si elle n'est pas nouvelle (Flon, 2012), acquiert ici une teneur particulière : quel est le statut 
de ces images? Que permettent-elles d'apprendre? De ressentir? Dans quel contexte sont-elles censées être utilisées ? En somme comment font-elle médiation?

Une autre génération de dispositifs apparait avec la mise en ligne de sites Internet et la production de dispositifs éditoriaux destinés à être consultés avant et/ou après la visite, voire même en dehors de la visite. En 1994, le site Internet indépendant weblouvre développé par un jeune ingénieur est précurseur de la mise en place par les grands musées, notamment Le Louvre et le musée d'Orsay, de plateformes qui dans un premier temps sont plutôt des « vitrines » des musées eux-mêmes (Schaeffer et Thierry, 2011) puis deviendront les outils de véritables stratégies de communication. Peu de temps après sont édités les premiers CD-Roms, dont le jeu Versailles 1685 : Complot à la Cour du Roi Soleil, et sont lancés des services en ligne, dont la Réunion des musées nationaux qui lance en 1996 le premier système de réservation de billets en ligne, puis la vente de produits à distance. Ces dispositifs sont soutenus par la Direction des musées de France et s'inscrivent dans le plan d'action gouvernemental pour la société de l'information. D'un point de vue éditorial, ces objets sont des produits culturels et ils interrogent le sens des pratiques culturelles (Davallon et Le Marec, 2000). On parle d' «interactifs» pour qualifier cette relation qui ménage à l'utilisateur une place toute particulière : mais quelle est la nature de cette interaction? Quel est le sens que donnent les visiteurs, les internautes, à ces consultations?

7 Dans les années 2000, de nouveaux dispositifs investissent l'enceinte des musées et offrent des services de personnalisation et de guidage de la visite. Ces outils apparaissent concomitamment à une réflexion sur l'expérience de visite et le statut du visiteur. À la fois, des études comme celles de Véron et Levasseur $(1983)$ ou de Wolf $(1980,1986)$ ont permis de porter l'attention sur la grande plasticité des interprétations des visiteurs, en d'autres termes, ces études ont montré qu'il existait autant de parcours de visite que de visiteurs. D'autre part, les années 2000 correspondent à la montée en puissance des logiques marketing dans le monde muséal (Le Marec et Chaumier, 2009) qui fragmente le public et propose de parler de cibles spécifiques. Ces années voient apparaître des dispositifs comme "Visite+ ", précurseur des outils d'accompagnement à la visite et de personnalisation des parcours des visiteurs, à la Cité des Sciences. Bientôt, des structures comme le MuséoLab d'Érasme, à Lyon, ou la plateforme MuseumLab du musée du Louvre, à Tokyo, proposent des expérimentations de guidage qui s'adaptent au profil du visiteur (sa langue, son choix de déambulation...). Avec l'usage des réseaux sociaux numériques, tels que Facebook ou Twitter, le musée interroge le lien qui l'unit à une nouvelle figure de public, son public en ligne et son réseau ${ }^{4}$. En 2008, les Abattoirs de Toulouse sont les premiers à créer une page Facebook. Une autre institution, le muséum d'Histoire naturelle de Toulouse se tourne dès 2008 vers la plateforme Wikimédia avec le projet «Phoebus » qui fait participer des amateurs à la prise de photographies des fonds des collections qui sont ensuite publiées sous licence libre sur Wikipédia. Les notions de participation commencent à apparaître et laissent à penser que ces outils permettent l'exercice d'une forme renouvelée de démocratie culturelle, comme en fait l'analyse critique Sarah Labelle (2008). 
Accès au Cyber-carnet proposé par Visite+ pour la post visite sur Internet.

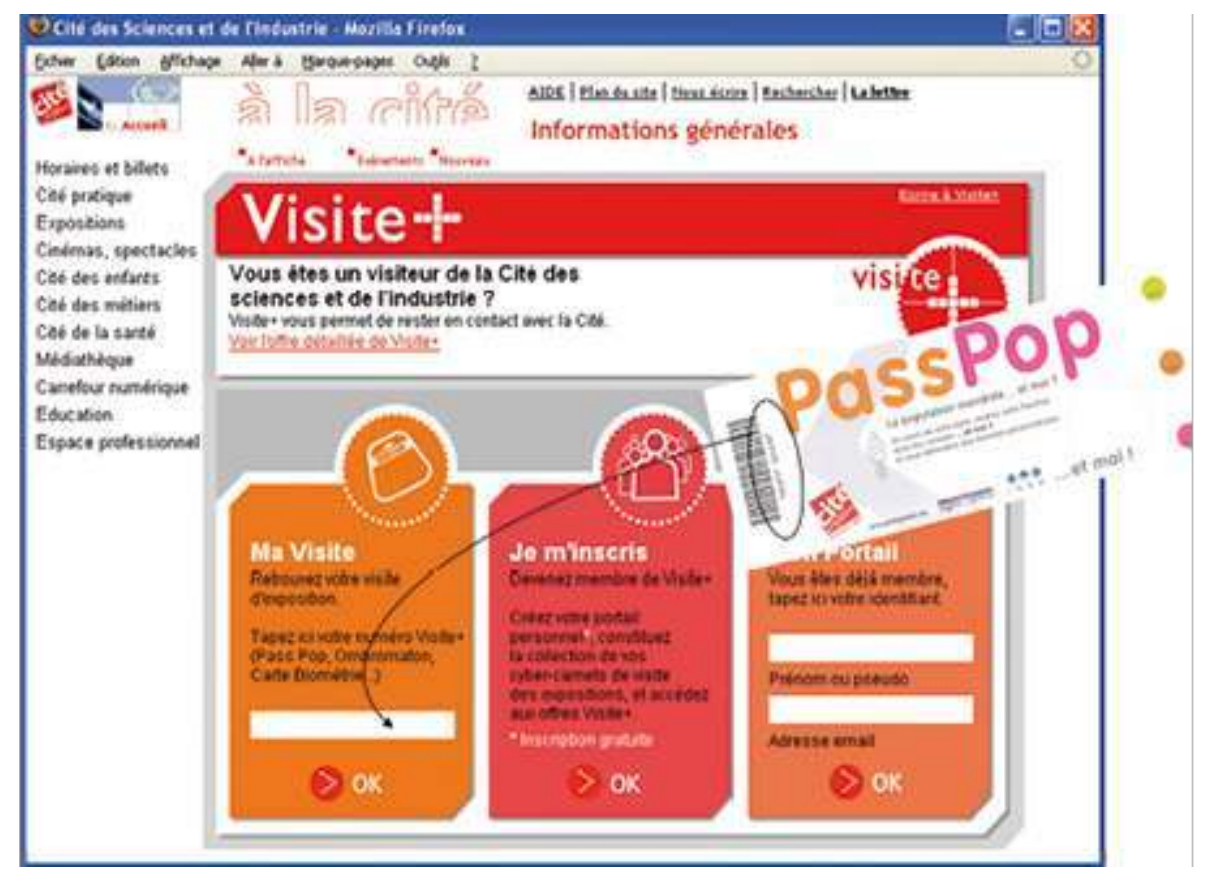

(c) CSI

\section{Un exemple : l'enjeu de l'accès au prisme des dispositifs numériques en ligne}

Attardons-nous sur l'un des enjeux soulevés par les relations entre publics, institutions muséales et dispositifs numériques, celui de l'accès et des publics en ligne.

Si l'on sait que la fréquentation des musées augmente depuis un certain nombre d'années ${ }^{5}$ , la fréquentation des sites Internet de musées explose littéralement et dépasse, pour les grandes institutions, bien largement la fréquentation physique. En 2012, le musée du Louvre enregistrait 11,4 millions de visiteurs uniques sur son site Internet, soit une progression de $8 \%$ en un an (2011/2012), quand la fréquentation physique était de 8,5 millions de visiteurs. 
Fréquentation des sites Internet des quatre premiers musées de France (millions de visites

\begin{tabular}{|lccc|}
\hline SITE & 2011 & 2012 & $\begin{array}{l}\text { Evolution } \\
2012 / 2011\end{array}$ \\
\hline www.louvre.fr & 10,6 & 11,4 & $+8 \%$ \\
www.chateauversailles.fr & 7,8 & 8,5 & $+8 \%$ \\
www.centrepompidou.fr & 5,5 & 5,9 & $+7 \%$ \\
www.musee-orsay.fr & 8,1 & 10,1 & $+25 \%$ \\
\hline
\end{tabular}

Sources : Rapports d'activité des musées/DEPS, 2013

10 Qui sont ces publics en ligne? Quelle est la relation qu'ils développent au musée ? Les utilisateurs des dispositifs numériques en ligne sont-ils amenés à devenir des visiteurs réels ? Comment organisent-ils la complémentarité ou la substituabilité entre les deux ? Olivier Donnat a montré que la probabilité d'être internaute augmentait régulièrement avec le niveau de participation aux pratiques culturelles traditionnelles (Donnat, 2008). Plus récemment, les études d'Anne Krebs montrent que les internautes vivant en France et s'intéressant aux visites virtuelles de musées ou d'expositions sont globalement de même profil socio-économique que les visiteurs « réels » de musées (Caro et Krebs, 2011). Nathalie Dutardre a montré, quant à elle, qu'il existait deux profils différents de public de site de musées en ligne, ceux qui souhaitent satisfaire un besoin de culture et de découverte, et ceux qui souhaitent préparer une visite du musée (Dutardre, 2003). Presque 10 ans plus tard, l'étude du CREDOC (Centre de recherche pour l'étude et l'observation des conditions de vie) sur l'usage des sites de musées montre que parmi les $35 \%$ de la population française qui utilisent Internet en lien avec les musées, les deux principaux usages de ces plateformes se distinguent de la même façon: $28 \%$ de la population y ont, au cours des douze derniers mois, recherché des informations pratiques (horaires, tarifs, accès...), et $16 \%$ ont effectué une visite virtuelle sur Internet d'une exposition, d'un musée ou d'un monument, (CREDOC, 2012). 


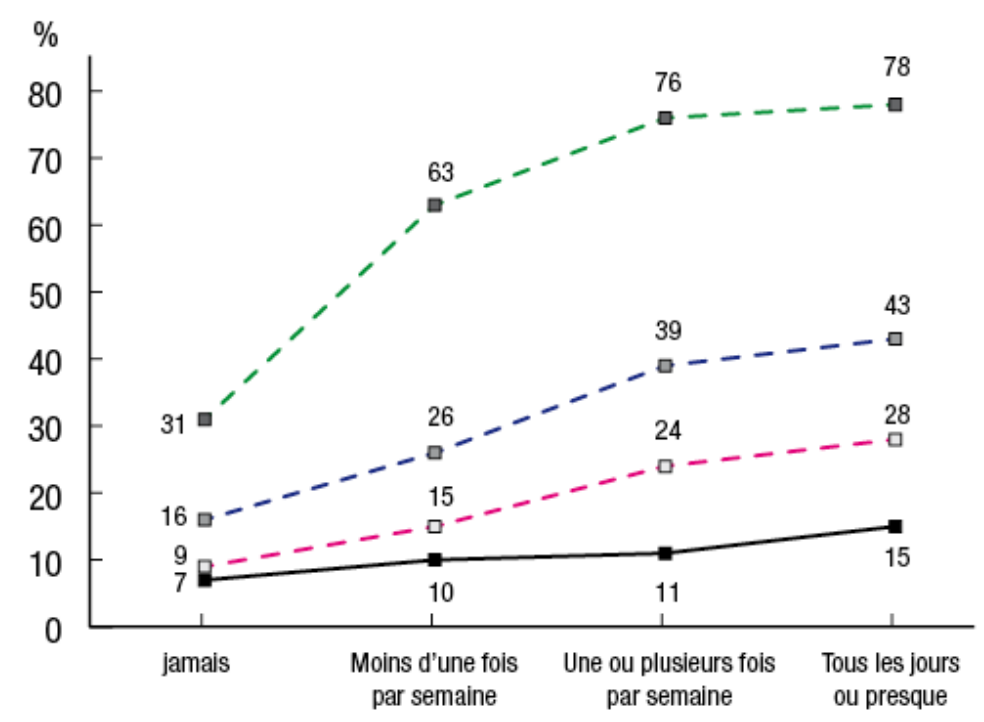

Sur 100 personnes de chaque groupe

Au cours des douzes derniers mois

- - - - sont allées au cinéma

sont allées au théâtre

- - - - sont allées au musée

ont lu 25 livres ou plus

Sources : Pratiques culturelles, Enquête 2008, DEPS, 2009.

11 Une autre question consiste à se demander comment on peut qualifier ces pratiques de consultation en ligne. Comment peut-on nommer ces publics? Quelles sont les formes d'être ensemble qui émergent de ces espaces numériques et en ligne ? Peut-on parler de communauté ? Repensons à la proposition de Bernard Rieder qui exhorte à la prudence et utilise la notion d'«écume" pour analyser cette sociabilitê à faible stabilitê (Rieder, 2010). Comment les internautes sont-ils impliqués par l'institution? Peut-on être ami avec un musée ? À quoi cela engage t'il ? Qu'est-ce que ça veut dire d'être «fan »? Cela veut-il dire érudit, conquis? Les débats font rage dans le monde professionnel des « muséogeeks » dont certains estiment que rien n'est plus simple que de cliquer une fois sur «J'aime », sur une page Facebook, sans porter attention aux contenus diffusés par la page ensuite. Mais alors que proposent les institutions muséales comme forme de participation lorsqu'elles offrent aux internautes la possibilité de «réagir » sur les réseaux sociaux ou sur leur site Internet? Est-ce que ce sont des pratiques d'écriture ou de lecture? Remplir des champs est bien une lecture au sens où les internautes reconnaissent dans le discours la place qui leur a été désignée par les énonciateurs. L'espace d'inscription de cette parole semble cependant toujours externe à l'espace où s'inscrit le discours muséal proprement dit (Le Marec, 2007).

\section{Conclusion : les formes d'enquête sur la relation entre le numérique et le musée}

12 La question qui reste en suspens est celle de savoir comment le chercheur ou le professionnel désireux de comprendre la relation entre publics en ligne et institution 
peut s'y prendre pour construire son enquête et ses outils de mesure. Il devra tout d'abord se demander quelle est la question qu'il adresse à cette relation : de quoi est-elle représentative ? De quoi est-elle censée témoigner ? Enfin quelle est la représentation que l'on se fait de ce public en ligne?

Si l'enquête est construite dans un objectif de comptage, alors c'est que le public en ligne est considéré comme un indicateur de la présence des musées sur la toile. Mais dans ce cas, quel est le périmètre du comptage : le nombre de «clics », les primo-visiteurs versus les multi-visiteurs, le nombre de pages lues, les circuits d'utilisation?

Si l'enquête est construite comme une évaluation, alors c'est que le public est considéré comme un utilisateur. Dans ce cas, il faut se demander comment ces utilisateurs prennent en main l'objet? Comment peut-on évaluer des logiques ergonomiques, des logiques de trajet sur des sites, sur des réseaux, entre des sites et des réseaux ?

Enfin, si l'enquête est considérée comme la compréhension d'une expérience culturelle, alors c'est que ce public est considéré comme un individu qui interprète une proposition de médiation. Dans ce cas, il faudra alors comprendre, par l'observation et l'entretien, en quoi la consultation d'un site dit quelque chose de la construction d'une relation à la culture et à l'institution culturelle.

\section{BIBLIOGRAPHIE}

Brochu D., Davallon, J., Camirand, C., Gottesdiener, H., Le Marec, J., Lemieux, A., Poli ,M.-S. et Tari K. Les musées face à l'édition mutlimédia. Dijon : OCIM, 1999.

Candito, N. Le numérique et ses effets sur la pratique d'évaluation, in Daignault, L. et Schiele, B, (dir) Les musées et leurs publics, savoirs et enjeux. Presses de l'université du Québec, 2014.

Caro, F., Krebs, A. et Evrard, Y. Analysing two modes of access to Art museum: the real/virtual orientation scale, 11th International Conference on Arts and Cultural Management (AIMAC 2011), Antwerp, Belgium, 3-6 July 2011.

Coutant, A. et Stenger, T. Processus identitaire et ordre de l'interaction sur les réseaux socionumériques, in Les enjeux de l'information et de la communication (en ligne), 2010.

CREDOC, enquête Conditions de vie et Aspirations, 2012.

Desprès-Lonnet, M, L'écriture numérique du patrimoine, de l'inventaire à l'exposition, Culture et Musée, $\mathrm{n}^{\circ}$ 14, 2009, pp. 19-38.

Donnat, O. Les pratiques culturelles des Français à l'ère numérique : enquête 2008. Paris : La Découverte / Ministère de la Culture et de la Communication, 2009.

Dutardre, N. Publics des musées en ligne et publics des musées réels : quels liens ? Paris :

Ministère de la Culture et de la Communication, Direction des musées de France, 2003.

Flon, É. Les mises en scène du patrimoine : savoir, fiction et médiation. Paris : Hermès Lavoisier, Paris, 2012. 
Jacobi, D. Images originales et images de reproduction dans l'exposition, in Jacobi, D. et Lochot, S. (dir.) Images d'exposition - Expositions d'images. Dijon : OCIM, 2005.

Jeanneret, Y. Y a-t-il (vraiment) des technologies de l'information ? Lille : Presses universitaires du Septentrion, 2011.

Le Deuff, $\mathrm{O}$. Monstres, légendes et hérauts : quelles pistes face à la tératogenèse documentaire ? ArchiveSIC, 2007.

Le Marec, J. et Chaumier, S. Évaluation muséale : Hermès ou les contraintes de la richesse, La Lettre de l'OCIM, n 126, novembre-décembre 2009, pp. 7-14.

Proulx, S. et Sénécal, M. L'interactivité technique, simulacre d'interaction sociale et de démocratie ? Technologies de l'information et Société, vol. 7, 1995, pp. 239-255.

Rieder, B. De la communauté à l'écume : quels concepts de sociabilité pour le « web social »? Tic\&société, vol. 4, $n^{\circ} 1$, mis en ligne le 17 mai 2010.

Sandri, É. De l'utilisation du terme « révolution » dans les technologies de l'information et de la communication : le cas des nouvelles technologies au musée, Métamorphoses et Bouleversements, Journées d'études doctorants et jeunes chercheurs, Laboratoire ICTT, 2012. Schafer V. et Benjamin, T. Le mariage de raison du musée d'art et du Web., Hermès, La Revue, $n$ ०6, 2011, pp. 102-105.

Sfez, L. Critique de la communication. Paris : Le Seuil, coll. « Points », 1992.

Topalian R. et Le Marec J. Visite+ : innover dans l'interactivité,

La Lettre de l'OCIM, n 118, juillet-août 2008, pp. 22-32.

Véron, E. et Levasseur, M. Ethnographie d'une exposition - L'espace, le corps et le sens. BPI Centre Georges Pompidou, 1983.

Wievorka, M. L'impératif numérique, ou La nouvelle ère des sciences humaines et sociales. Paris: CNRS, 2013.

Wolf, R. A Naturalistic View of Evaluation, Museum News, n 58, 1980, pp. 39-45.

Wolf, R. The Missing link: a look at the role of orientation in enriching the museum experience, Journal of Museum Education: Roundtable Reports, n 11, 1986, pp. 17-21.

\section{NOTES}

1. On pense notamment à l'appel à projet "Services numériques culturels innovants" lancé en 2010 par le ministère de la Culture, www.culturecommunication.gouv.fr/Politiquesministerielles/Recherche-Enseignement-superieur-Technologies/Innovation-numerique/ Services-numeriques-culturels-innovants

2. Extrait d'une plaquette de présentation du salon consacré aux musées, lieux de culture et tourisme SIME SITEM, 2014, www.museumexperts.com/simesitem/salon/

3. La convention de 1972 de l'UNESCO pour la protection du patrimoine mondial, naturel et culturel définit le patrimoine culturel à l'article premier. Le patrimoine culturel comprend : "Les monuments : œuvres architecturales, de sculpture ou de peinture monumentales, éléments ou structures de caractère archéologique, inscriptions, grottes et groupes d'éléments, qui ont une valeur universelle exceptionnelle du point de vue de l'histoire, de l'art ou de la science; Les ensembles : groupes de constructions isolées ou réunies, qui, en raison de leur architecture, de leur unité, ou de leur intégration dans le paysage, ont une valeur universelle exceptionnelle du point de vue de l'histoire, de l'art ou de la 
science; Les sites : œuvres de l'homme ou œuvres conjuguées de l'homme et de la nature, ainsi que les zones y compris les sites archéologiques qui ont une valeur universelle exceptionnelle du point de vue historique, esthétique, ethnologique ou anthropologique". http://whc.unesco.org/fr/conventiontexte/

4. Pour rappel, "les réseaux sociaux numériques constituent des services web qui permettent aux individus : (1) de construire un profil public ou semi-public au sein d'un système, (2) de gérer une liste des utilisateurs avec lesquels ils partagent un lien, (3) de voir et naviguer sur leur liste de liens et sur ceux établis par les autres au sein du système, et (4) fondent leur attractivité essentiellement sur les trois premiers points et non sur une activité particulière." (Coutant et Stenger, 2010).

5. D'après les statistiques fournies par le ministère de la Culture et de la Communication, la fréquentation des musées de France est passée de 45,3 millions de visiteurs en 2005 à 61,6 millions en 2012.

\section{RÉSUMÉS}

Après avoir rappelé que chaque génération de dispositifs numériques a façonné une relation particulière entre l'institution muséale et ses publics, l'auteur s'attache plus précisément à montrer comment l'enjeu de l'accès au musée - notamment de nouveaux publics - est au cœur de cette problématique et comment le numérique procure à ses utilisateurs une expérience culturelle spécifique.

\section{INDEX}

Mots-clés : musée, dispositif numérique, institution culturelle

\section{AUTEUR}

\section{CAMILLE JUTANT}

maître de conférences en Science de l'Information et de la Communication, laboratoire ELICO, université de Lyon 2 camillejutant@hotmail.com 